\title{
Refractory pseudomembranous colitis that was treated successfully with colonoscopic fecal microbial transplantation
}

\author{
Jun Young Shin, Eun Jung Ko, Seung Ho Lee, Jong Bum Shin, Shin Il Kim, Kye Sook Kwon, Hyung Gil Kim, \\ Yong Woon Shin, Byoung Wook Bang \\ Division of Gastroenterology, Department of Internal Medicine, Inha University School of Medicine, Incheon, Korea
}

\begin{abstract}
Pseudomembranous colitis (PMC) is a nosocomial and opportunistic infection caused by Clostridium difficile. PMC is related to the use of antibiotics leading to intestinal dysbiosis and an overgrowth of $C$. difficile. Metronidazole or vancomycin is considered to be the standard therapy for the management of PMC. However, PMC has a $15 \%-30 \%$ recurrence rate and can be refractory to standard treatments, resulting in morbidity and mortality. Here we describe a patient who experienced refractory PMC who was treated with fecal microbiota transplantation. A 69-year-old woman was admitted to the hospital with consistent abdominal pain and diarrhea, which had been present for 5 months. She was diagnosed with PMC by colonoscopy and tested positive for $C$. difficile toxin. Even though she took metronidazole for 10 days, followed by vancomycin for 4 weeks, her symptoms did not improve. Because of her recurrent and refractory symptoms, we decided to perform fecal microbiota transplantation. Fifty grams of fresh feces from a donor were obtained on the day of the procedure, mixed with $500 \mathrm{~mL}$ of normal saline, and then filtered. The filtered solution was administered to the patient's colon using a colonoscope. After the procedure, her symptoms rapidly improved and a follow-up colonoscopy showed that the PMC had resolved without recurrence. (Intest Res 2016;14:83-88)
\end{abstract}

Key Words: Clostridium difficile; Fecal microbial transplantation; Colonoscopy; Enterocolitis, pseudomembranous

\section{INTRODUCTION}

Pseudomembranous colitis (PMC) is a nosocomial infection mainly caused by the use of antibiotics and is commonly associated with abdominal pain and diarrhea. A gradual increase in PMC incidence has been observed, coincident with increasing antibiotic consumption..$^{1-3}$ Despite the use of antibiotics for the primary management of PMC, it has a re-

Received December 8, 2014. Revised March 10, 2015

Accepted March 16, 2015

Correspondence to Byoung Wook Bang, Division of Gastroenterology,

Department of Internal Medicine, Inha University College of Medicine, 27

Inhang-ro, Jung-gu, Incheon 22332, Korea. Tel: +82-32-890-2548, Fax:

+82-32-890-2549, E-mail: bangbu@inha.ac.kr

Financial support: This study was supported by the Inha University

research fund. Conflict of interest: None. currence rate of $15 \%-30 \%$ and patients who had one recurrence can reach up to $40 \%$ of a second recurrence. ${ }^{4}$ This high rate of recurrent and refractory PMC not only lowers the quality of patients' lives, but also may predispose patients to severe megacolon, perforation, shock, and other complications. $^{5-7}$

Fecal microbiota transplant (FMT) has emerged in recent years as a treatment option for patients with recurrent and antibiotic-refractory PMC, and many studies have achieved favorable results with this procedure. ${ }^{8-15}$ Here we report a case of refractory PMC which was successfully resolved without recurrence after performing FMT using a colonoscope.

\footnotetext{
๑ Copyright 2016. Korean Association for the Study of Intestinal Diseases. All rights reserved.

This is an Open Access article distributed under the terms of the Creative Commons Attribution Non-Commercial License (http://creativecommons.org/licenses/by-nc/4.0)

which permits unrestricted non-commercial use, distribution, and reproduction in any medium, provided the original work is properly cited.
} 


\section{CASE REPORT}

A 69-year old woman was admitted to the Inha University Hospital with complaints of mucous diarrhea and low abdominal pain with repeated relapses and remission of symptoms over 5 months. She was taking medication for hypertension and had a history of adjuvant chemotherapy after undergoing a low anterior resection due to rectal cancer 12 years prior to admission to our hospital. Five months ago, the patient was admitted to a local hospital due to diarrhea that developed after eating pork. She then visited our hospital's outpatient unit because her symptoms remained the same. Even though antidiarrheals and probiotics were administered to the patient in the outpatient unit, her symptoms were not improved.

A colonoscopy performed two months prior to her visit revealed edema, hyperemia, and multiple whitish plaques from the transverse colon to the sigmoid colon (Fig. 1A, B). Since pathologic findings included denuded epithelium covered with a mucoid exudate (Fig. 1C) and stool test results were positive for Clostridium difficile toxin, she was diagnosed with PMC. Her CRP level was elevated to 7.99 $\mathrm{mg} / \mathrm{dL}$ and no other bacteria were detected from blood or stool cultures. Administration of metronidazole (500 mg), orally 3 times per day for 10 days, temporarily improved her symptoms, with a decrease of CRP to $1.58 \mathrm{mg} / \mathrm{dL}$. However, diarrhea persisted and her symptoms were gradually worsened. Subsequently, vancomycin (250 mg) was given orally 4 times per day for 2 weeks, and symptoms were improved for a week, but diarrhea persisted.

She underwent colonoscopy again after being admitted to our hospital 1 month prior to her final admission for FMT.
Colonoscopy revealed PMC, and re-elevated CRP levels $(8.82 \mathrm{mg} / \mathrm{dL})$. Hence, a daily dose of oral vancomycin was increased to $500 \mathrm{mg}$, 4 times per day for 2 weeks. However, mucous diarrhea persisted after discharge and the symptoms were not improved. Since her PMC was determined to be refractory to antibiotics, we decided to perform an FMT after obtaining consent from the patient and her guardian. At the time of admission for FMT, she had stable vital signs (blood pressure 146/78 $\mathrm{mmHg}$, heart rate 80 beats/min, respiratory rate 18 breaths $/ \mathrm{min}$, and a temperature of $36.0^{\circ} \mathrm{C}$ ) but was critically ill. She complained of systemic muscle weakness, abdominal pain and a feeling of incomplete defecation. The patient suffered from diarrhea occurring 5-8 times per days. Mild tenderness in the lower left abdomen was detected in a physical exam. Her heart and breath sounds were normal and skin rash was not observed.

Laboratory tests revealed WBC $8,860 / \mathrm{mm}^{3}$, hemoglobin $13.3 \mathrm{gm} / \mathrm{dL}$, platelet count $190,000 / \mathrm{mm}^{3}$, total protein 6.9 gm/dL, albumin $3.8 \mathrm{gm} / \mathrm{dL}$, total bilirubin $1.3 \mathrm{mg} / \mathrm{dL}$, AST 19 IU/L, ALT $15 \mathrm{IU} / \mathrm{L}$, ALP $161 \mathrm{IU} / \mathrm{L}$, BUN 9.4 mg/dL, creatinine $0.87 \mathrm{mg} / \mathrm{dL}, \mathrm{CRP} 0.21 \mathrm{mg} / \mathrm{dL}$, Na $138 \mathrm{mEq} / \mathrm{L}, \mathrm{K} 2.9 \mathrm{mEq} / \mathrm{L}$ and $\mathrm{Cl} 105 \mathrm{mEq} / \mathrm{L}$. Based on these results, she was suspected to have hypokalemia attributable to repeated diarrhea. No specific findings were observed on simple chest radiographs.

The patient's daughter, who had no underlying diseases or gastrointestinal (GI) symptoms, volunteered to be the donor. In addition, the daughter had no history of medication over the previous 3 months. Donor screening tests were carried out, including a parasite exam, stool culture, and a susceptibility test for Salmonella, Shigella, and Campylobacter species, a $C$. difficile toxin A/B test. In addition, several serum tests were done, including serum Ameobic Antibody, HAV
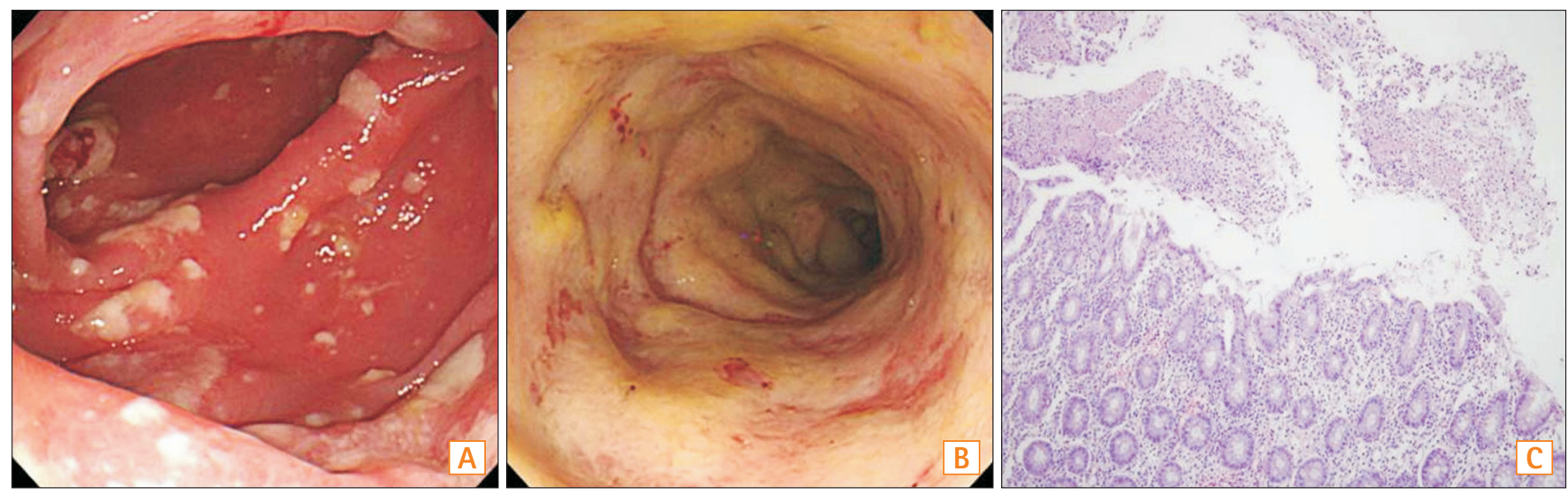

Fig. 1. Initial colonoscopic and pathologic findings. Colonoscopy showed multiple whitish patches on the mucosa in the transverse colon (A) and diffuse hyperemic and edematous mucosa with whitish plaques in the sigmoid colon (B). (C) Pathologic finding revealed denuded epithelium covered with inflammatory debris and neutrophils ( $\mathrm{HEE}, \times 40)$. 
IgG/M, HBsAg/Ab, HBcAb, HCV Ab, HIV Ag/Ab, vereneal disease research laboratory. The donor had no abnormal findings. Before the transplantation, the use of antibiotics was suspended and bowel cleansing was performed. Colonoscopy revealed edematous and hyperemic mucosal changes with multiple whitish patches from the transverse colon to the sigmoid colon (Fig. 2A, B).

On the day of the procedure, $50 \mathrm{~g}$ of fresh feces were collected from the donor and then mixed and stirred with $500 \mathrm{~mL}$ of normal saline. Subsequently, the supernatant of the solution was filtered using a coffee filter or gauze (Fig. 2C). Approximately $500 \mathrm{~mL}$ of the filtered fecal suspension was administered into the proximal ascending colon via a colonoscope (Fig. 2D). After the FMT there were no complications and the patient was discharged 2 days after the procedure. At the first week follow-up visit, mild abdominal discomfort was present but diarrhea was improved. One month after being discharged, a follow-up colonoscopy was performed and found no relapsing symptoms. Mucosal edema and hyperemia were also dramatically improved (Fig. 3). The patient is currently being monitored and had no recurrence of symptoms at the last follow-up, 9 months after the procedure.

\section{DISCUSSION}

C. difficile infection is one of the common nosocomial infections and the leading cause of antibiotic-associated diarrhea. Intestinal dysbiosis caused by antibiotic use can eventually lead to proliferation of $C$. difficile, a gram-positive anaerobic bacteria, and toxin production and can result in symptoms such as abdominal pain and diarrhea. PMC is diagnosed based on a positive result for $C$. difficile toxin or characteristic findings during colonoscopy. ${ }^{1,5,16,17}$ For primary therapy of PMC, oral metronidazole $(500 \mathrm{mg})$ is administered 3 times per day for 10-14 days. Oral vancomycin (125-250 mg) is recommended 4 times per day for 10-14 days for severe cases. ${ }^{7,8,18}$ Despite favorable treatment outcomes, PMC has a $15 \%-30 \%$ recurrence rate and repeated relapses in the course of the disease reduce the response to treatment. ${ }^{1,4}$ At first recurrence, the initial therapy can be implemented again, depending on a re-evaluating the severity of PMC. In cases of repeated recurrence, oral vancomycin (500 mg, 4 times per day) is considered and intravenous
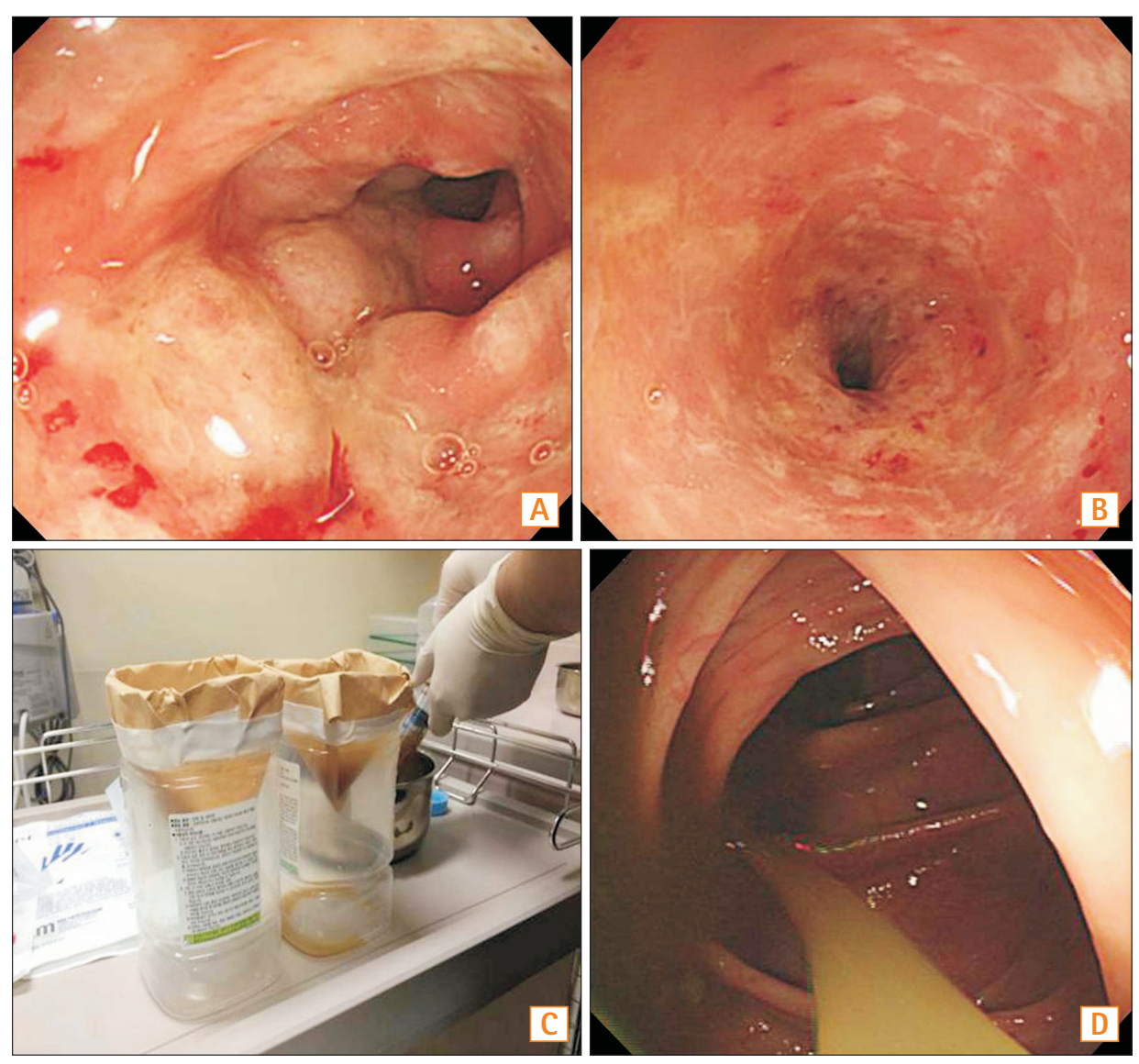

Fig. 2. Colonoscopic findings on the day of the procedure and fecal microbial transplantation. Colonoscopy revealed edematous and hyperemic mucosal changes with multiple whitish patches from the transverse colon to the rectum ( $A$ : descending colon, B: sigmoid colon). (C) $50 \mathrm{~g}$ of fresh feces were collected from the donor and then mixed and stirred with $500 \mathrm{~mL}$ of normal saline. That solution was then filtered using a coffee filter and/or gauze. (D) The filtered fecal solution was administered into the proximal ascending colon via a colonoscope. 


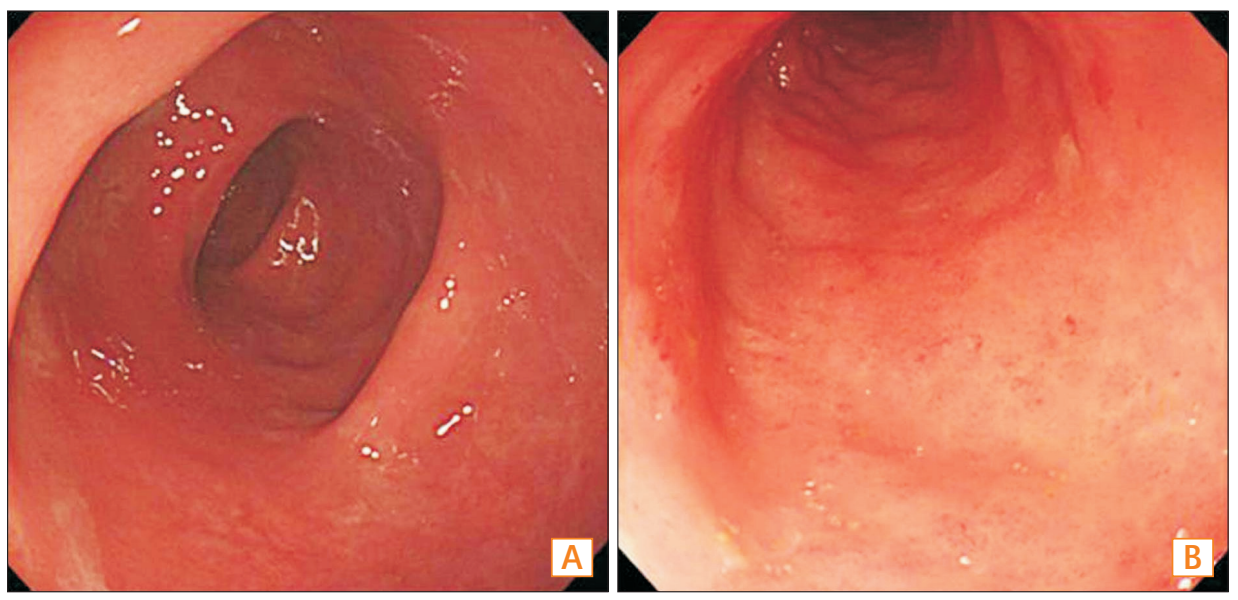

Fig. 3. Colonoscopic findings one month after fecal microbial transplantation. Compared to the previous colonoscopy in this patient, mucosal edema and hyperemia was dramatically improved (A: sigmoid colon, B: rectum). administration of metronidazole or a vancomycin enema is recommended, in combination with oral vancomycin or alone. ${ }^{16,17}$ However, recurrent and refractory PMC is more difficult to treat, ${ }^{1,6,8}$ and is usually associated with megacolon, perforation, shock, and other severe complications related to the increasing incidence of PMC. ${ }^{7}$ Although the use of metronidazole failed in the case reported here, the patient was diagnosed with mild PMC due to the absence of high fever or leukocytosis. Her symptoms persisted despite the use of vancomycin ( $250 \mathrm{mg}$, 4 times per day for 2 weeks). Since the initial dose of vancomycin was determined to be insufficient, it was increased to $500 \mathrm{mg}$, 4 times per day for another 2 weeks. However, an FMT was planned after the patient's PMC was diagnosed as refractory.

New therapeutic approaches have been investigated for the treatment of recurrent and refractory PMC, and a large number of recent studies have explored the use of FMT. ${ }^{8-15}$ The concept of an FMT was first reported in 1958 by Eiseman et al. ${ }^{9}$ for the treatment of fulminant PMC. Since then, a considerable number of case reports and studies on the treatment's effects and methodologies have been performed worldwide. In a recent randomized control trial, FMT had a better outcome than the use of vancomycin in patients with recurrent and refractory PMC. ${ }^{8}$ A domestic study also reported a successful case of treating recurrent and refractory PMC via enema ${ }^{11}$ and upper GI endoscopy. ${ }^{12}$ This case report was meaningful in that it compared clinical data and colonoscopic findings from follow-up colonoscopies in cases where PMC was managed with colonoscopy.

An FMT can be performed via a nasogastric tube, upper endoscopy, enema, colonoscopy, and other methods. ${ }^{18} \mathrm{Al}-$ though some concerns have been raised with regard to the re-establishment of the normal intestinal flora from transplanted microbiota in the use of a nasogastric tube or upper endoscopy, studies have not shown significant differences in these methods. However, there may be problems such as vomiting, the risk of aspiration, and patient's rejection of the transplant. In our hospital, there was one case in which aspiration pneumonia developed in a patient with cerebrovascular disease after undergoing fecal transplantation with a nasogastric tube. The shortcomings of colonoscopy are that it is relatively more complicated than nasogastric tube insertion or upper GI endoscopy, and bowel cleansing is essential for proper examination. The advantages are that vomitingrelated problems can be excluded, unnecessary FMT can be avoided, and transplantation can be done onto the lesion site by examining the mucosal state with colonoscopy. When the fecal solution can be retained in the colon for a sufficient time after transplantation, fecal transplant via colonoscopy is anticipated to be an effective method for treating PMC. Even though performing an enema would have been an easier procedure in this case, we chose transplantation via colonoscopy due to the short transit time. Furthermore, instead of injecting into the transverse or sigmoid colon, the fecal suspension was injected into the ascending colon for a longer colonic transit time. After transplantation, the patient was encouraged to rest in a supine position and to retain her stool, if possible. Although the side effects of FMT via colonoscopy are known to be minimal, ${ }^{19}$ cautions should be taken in using colonoscopy in patients with severe PMC associated with paralytic ileus or megacolon, due to the risk of complications such as perforations.

Successful treatment of FMT cases has been reported and several multicenter randomized controlled studies and meta-analyses have demonstrated the favorable results of this procedure. However, FMT has not been widely implemented in Korea because of patients being unwilling to undergo fecal transplantation and concerns about infections. ${ }^{20}$ 
In the future, patients will hopefully accept and understand the benefits of fecal transplantation, even though it may be aesthetically unappealing, through sufficient explanation during interviews by clinicians. Moreover, a donor screening test is warranted to prevent secondary infections caused by the transplantation. In this case report, the patient was compliant when the need for the procedure and the clinical outcomes of FMT were fully explained. Secondary infection was prevented as the patient's daughter was chosen as the donor and blood and fecal screening tests were thoroughly conducted before the procedure.

Bowel cleansing was performed in preparation for the FMT, as described in previous studies. ${ }^{8,10,13}$ On the day of procedure, $50 \mathrm{~g}$ of fresh feces were collected from the donor and then mixed and stirred with normal saline. Subsequently, the supernatant of the solution was filtered using a coffee filter or gauze (Fig. 2C). By repeating the above process, approximately $500 \mathrm{~mL}$ of fecal suspension were prepared and injected to the patient's colon via colonoscopy. During this process, the coffee filter takes longer to filter out fecal particles than gauze since the filter is blocked by those particles. Even though the effectiveness of FMT has been demonstrated, the transplantation procedure has not yet been standardized. ${ }^{15,20}$ According to a systematic review by Gough et al. ${ }^{15}$ in 2011, no differences were found in the rate of successful treatment in relation to the amount of used stool. However, a significantly lower recurrence rate was observed in the group transplanted with more than $50 \mathrm{~g}$ of stool (1\%) compared to the group transplanted with less than $50 \mathrm{~g}(4 \%)$. Treatment rates showed a significant difference in cases transplanted with more than $500 \mathrm{~mL}$ of fecal solution compared with cases transplanted with less than $200 \mathrm{~mL}$ of fecal solution (97\% and $80 \%$, respectively). Based on the above results, we decided that preparing approximately $500 \mathrm{~mL}$ of fecal solution by mixing more than $50 \mathrm{~g}$ of feces with saline solution was appropriate for the transplantation.

Van Nood et al. ${ }^{8}$ selected donors through a questionnaire and screening tests among donor volunteers. Kelly et al. ${ }^{10}$ involved the patient's family members as donors. An insignificant difference was found in treatment success rates between the two groups ( $94 \%$ and $92 \%$, respectively) and severe side effects were not seen. These outcomes provide a basis for expanding donor selection through adequate screening tests. In this case report, the patient's daughter volunteered to be the donor. Transplantation from a family member is thought to alleviate the patients concerns towards fecal transplantation. However, the screening process took approximately one week before fecal transplantation was possible. In recent years, administration of freeze-dried fecal capsules from donors has lead to more timely and effective FMT, exhibiting successful results in over $90 \%$ of urgent-care patients with PMC. ${ }^{21}$

The patient in the current case report is currently being followed up and has been without recurrence for 9 months after the FMT. In a study on 94 patients followed up after FMT via enema, there were no cases of recurrence between 6-24 months. ${ }^{22}$ A previous study reported that eight relapsed cases out of 77 patients were attributable to antibiotic use, three months after fecal transplantation via colonoscopy. Recurrence was not detected in the remaining patients during a mean follow-up period of 17 months. ${ }^{23}$ Based on these results, no relapse of PMC is expected over the long-term in the current case report.

The above findings from several case reports and systematic reviews demonstrate the efficacy of FMT. The patient in the current case report showed a dramatic improvement after a single session of FMT. The procedure was simple and additional management was unnecessary after fecal transplantation. Although FMT has not been performed frequently in Korean medical institutions due to patient aversion to fecal transplantation and concerns about infection, FMT is now officially recommend in European treatment guidelines. ${ }^{24}$ FMT is expected to be the standard therapy for the management of recurrent and refractory PMC domestically in the future.

\section{REFERENCES}

1. Kelly CP, LaMont JT. Clostridium difficile - more difficult than ever. N Engl J Med 2008;359:1932-1940.

2. Lee YJ, Choi MG, Lim CH, et al. Change of Clostridium difficile colitis during recent 10 years in Korea. Korean J Gastroenterol 2010;55:169-174.

3. Lee JH, Lee SY, Kim YS, et al. The incidence and clinical features of Clostridium difficile infection; single center study. Korean J Gastroenterol 2010;55:175-182.

4. Seo GS. Clostridium difficile Infection: What's New? Intest Res 2013;11:1-13.

5. Wilcox MH. Descriptive study of intravenous immunoglobulin for the treatment of recurrent Clostridium difficile diarrhoea. J Antimicrob Chemother 2004;53:882-884.

6. Kelly CP. Fecal microbiota transplantation - an old therapy comes of age. N Engl J Med 2013;368:474-475.

7. Pepin J. Improving the treatment of Clostridium difficileassociated disease: where should we start? Clin Infect Dis 2006;43:553-555. 
8. van Nood E, Vrieze A, Nieuwdorp M, et al. Duodenal infusion of donor feces for recurrent Clostridium difficile. N Engl J Med 2013;368:407-415.

9. Eiseman B, Silen W, Bascom GS, Kauvar AJ. Fecal enema as an adjunct in the treatment of pseudomembranous enterocolitis. Surgery 1958;44:854-859.

10. Kelly CR, de Leon L, Jasutkar N. Fecal microbiota transplantation for relapsing Clostridium difficile infection in 26 patients: methodology and results. J Clin Gastroenterol 2012;46:145-149.

11. Moon KR, Sohn KM, Park BM, et al. Successful fecal transplantation by enema for recurrent and refractory Clostridium difficile infection. J Korean Geriatr Soc 2013;17:152-156.

12. Gweon TG, Choi MG, Lee SK, et al. Two cases of refractory pseudomembranous colitis that healed following fecal microbiota transplantation. Korean J Med 2013;84:395-399.

13. Burke KE, Lamont JT. Fecal transplantation for recurrent Clostridium difficile infection in older adults: a review. J Am Geriatr Soc 2013;61:1394-1398.

14. Yoon SS, Brandt LJ. Treatment of refractory/recurrent $C$. difficile-associated disease by donated stool transplanted via colonoscopy: a case series of 12 patients. J Clin Gastroenterol 2010;44:562-566.

15. Gough E, Shaikh H, Manges AR. Systematic review of intestinal microbiota transplantation (fecal bacteriotherapy) for recurrent Clostridium difficile infection. Clin Infect Dis 2011;53:9941002.

16. Cohen SH, Gerding DN, Johnson S, et al. Clinical practice guidelines for Clostridium difficile infection in adults: 2010 update by the society for healthcare epidemiology of America (SHEA) and the infectious diseases society of America (IDSA). Infect Control Hosp Epidemiol 2010;31:431-455.
17. Bauer MP, Kuijper EJ, van Dissel JT; European Society of Clinical Microbiology and Infectious Diseases. European Society of Clinical Microbiology and Infectious Diseases (ESCMID): treatment guidance document for Clostridium difficile infection (CDI). Clin Microbiol Infect 2009;15:1067-1079.

18. Fekety R, Silva J, Kauffman C, Buggy B, Deery HG. Treatment of antibiotic-associated Clostridium difficile colitis with oral vancomycin: comparison of two dosage regimens. Am J Med 1989;86:15-19.

19. Rohlke F, Stollman N. Fecal microbiota transplantation in relapsing Clostridium difficile infection. Therap Adv Gastroenterol 2012;5:403-420.

20. Kang J. Fecal Bacteriotherapy for Recurrent Clostridium difficile infection: a systemic literature review. Korean J Nosocomial Infect Control 2012;17:70-78.

21. Youngster I, Russell GH, Pindar C, Ziv-Baran T, Sauk J, Hohmann EL. Oral, capsulized, frozen fecal microbiota transplantation for relapsing Clostridium difficile infection. JAMA 2014;312:1772-1778.

22. Lee CH, Belanger JE, Kassam Z, et al. The outcome and longterm follow-up of 94 patients with recurrent and refractory Clostridium difficile infection using single to multiple fecal microbiota transplantation via retention enema. Eur J Clin Microbiol Infect Dis 2014;33:1425-1428.

23. Brandt LJ, Aroniadis OC, Mellow M, et al. Long-term follow-up of colonoscopic fecal microbiota transplant for recurrent Clostridium difficile infection. Am J Gastroenterol 2012;107:10791087.

24. Debast SB, Bauer MP, Kuijper EJ; European Society of Clinical Microbiology and Infectious Diseases. European Society of Clinical Microbiology and Infectious Diseases: update of the treatment guidance document for Clostridium difficile infection. Clin Microbiol Infect 2014;20(Suppl 2):1-26. 